\title{
Childhood Pilomyxoid Astrocytoma
}

National Cancer Institute

\section{Source}

National Cancer Institute. Childhood Pilomyxoid Astrocytoma. NCI Thesaurus. Code C114970.

A pilomyxoid astrocytoma that occurs during childhood. 